Steffen $W$. Hillebrecht und Martina Höhns?

\title{
Struktur und Organisation
} kirchlicher Kommunikation

Kommunikationsarbeit im Bistum

\section{Vorbemerkungen}

Kommunikations- und Öffentlichkeitsarbeit sind in jeder Institution und in jedem Unternehmen ein zentraler Bestandteil der täglichen Arbeit, besonders aber von strategischen Entscheidungen. ${ }^{2}$ Kommunikation betrifft alle Partner einer Einrichtung: Lieferanten und Kunden, Mitarbeiter und die Gesellschaft als ganze. Kommunikation umfaßt alle Bereiche, mit denen "das Unternehmen" in Kontakt mit seinen Partnern tritt, z.B. Öffentlichkeitsarbeit, Werbung, Marktforschung, Produktdesign (als Ausdruck eines bestimmten Qualitätsverständnisses), persönliche Betreuung und Kundendienst sowie die interne Kommunikation der einzelnen Abteilungen. Daher ist es wichtig, die Mitarbeiterinnen und Mitarbeiter zu kommunikativem Verhalten anzuleiten und sie in Kommunikationstechniken zu schulen.

Die einheitliche, effektive Gestaltung der Kommunikationsarbeit wird häufig in Richtlinien zur Corporate Identity zusammengefaßt, die für alle Mitarbeiterinnen und Mitarbeiter, ohne Ansehen der Funktionsebene, verbindlich sind. Entsprechende Fachabteilungen für Kommunikationsarbeit helfen der Unternehmensleitung wie auch den einzelnen Unternehmensbereichen bei der effizienten Planung, Koordination und Durchführung aller Kommunikationsmaßnahmen. ${ }^{3}$

Da "Communio" ein wesentliches Element der Kirche ist, erfordert die kirchliche Binnenkommunikation und das Gespräch der Kirche mit der Welt eine wohlstrukturierte, professionelle Kommunikationsarbeit. In Begleitung eines Projekts zur „Erarbeitung eines publizistischen Plans" in einer deutschen Diözese, bei dem eine Optimierung der Kommunikations- und Öffentlichkeitsarbeit im Bistum angestrebt wurde, haben die Zentralstelle Medien der Deutschen Bischofskonferenz und die MDG Medien-Dienstleistung GmbH eine „Arbeitshilfe zur

1 Die Autoren danken allen Ratgebern für ihre kritische Begleitung, ganz besonders Herrn Lic. theol. Thomas Hagenhoff (Bichöliches Generalvikariat Hildesheim).

2 Vgl. z.B. Peter Ulrich/Edgar Fluri: Management, 6. Aufl., Bern u.a.: P. Haupt 1992, S. 36f; 171ff; Günter Wohe: Einführung in die Allgmeine Betriebswirtschaftslehre, 16. Aufl., München: Vahlen 1986, S. $89 \mathrm{ff}$.

3 Vgl. Franz Bőcker: Marketing, 3. Aufl., Stuttgart: G. Fischer 1991, S. $357 \mathrm{ff.} \mathrm{Dietger}$ Hahn: Unternehmensfïhrung und Öffentlichkeitsarbeit, in: Zeitschrift für Betriebswirtschaft , 62. Jg. (1992), S. 137-157. Hans Raffee: Integrierte Kommunikation, in: Werbeforschung \& Praxis, 36. Jg., Nr. 3/1991, S. 87-90. 
Struktur und Organisation der Kommunikation im Bistum" 4 entwickelt. In dieser Arbeitshilfe werden die Ergebnisse und Erfahrungen des Projekts dokumentiert und Anregungen für die Öffentlichkeits- und Kommunikationsarbeit in einem Bistum vorgelegt. Die Arbeitshilfe orientiert sich an den Aussagen zu Aufgaben und Organisation kirchlicher Kommunikationsarbeit in den beiden grundlegenden römischkatholischen Dokumenten über Medien und Kommunikation "Communio et Progressio"5 und „Aetatis Novae"6. Erfolgsfaktoren der Unternehmenskommunikation werden jedoch ebenso berücksichtigt wie kirchliche Spezifika und Erfahrungen. Die Arbeitshilfe wird im nachfolgenden in ihren Kernpunkten wiedergegeben.

\section{Kirche als kommunikatives Ereignis}

Kommunikation gehört - im Sinne von Mitteilung und Gemeinschaft ${ }^{7}$ zum Wesen der Kirche. Von Anfang an haben Christen die verschiedensten Formen der Kommunikation genutzt, um das Evangelium zu verkündigen, Gemeinden aufzubauen und ihre Glaubenserfahrungen weiterzugeben. Der Auftrag der Kirche, die frohe Botschaft vom Reich Gottes öffentlich $\mathrm{zu}$ verkünden, ist nach wie vor gültig. Beständig geändert haben sich hingegen die Bedingungen und die Mittel der Kommunikation.

Die europäischen Bischöfe haben die Motivation für eine kirchliche Kommunikationsarbeit 1995 in Assisi folgendermaßen formuliert: "Die Kirche selbst ist von ihrem Wesen her auf Kommunikation angelegt, die zur Gemeinschaft (Communio) mit Gott und den Menschen führt. Die verschiedenen Formen der innerkirchlichen Kommunikation müssen deshalb der Förderung und Pflege dieser Communio und auch dem Austausch der Vielfalt innerhalb der Kirche dienen. Die Qualität der

4 Veroffentlicht als gemeinsames Arbeitsmaterial der MDG Medien-Dienstleistung GmbH, München und der Zentralstelle Medien der Deutschen Bischofskonferenz, Bonn. Die Arbeitshilfe ist gegen eine Schutzgebuthr bei der MDG und der Zentralstelle Medien erhăltlich.

5 Communio et Progressio (CeP), hrsg. von der Papstlichen Kommission für die Instrumente der sozialen Kommunikation vom 23. Mai 1971, 2. Aufl., Trier: Paulinus 1991.

6 Aetatis Novae (AN), hrsg. vom Pupstlichen Rat für die Sozialen Kommunikationsmittel, vom 22. Februar 1992 (= Arbeitshilfen Nr. 98 der Deutschen Bischofskonferenz, Bonn 1992).

7 Vgl. Josef Kardinal Ratzinger; Alberto Bovone: Kirche als Communio, in: HerderKorrespondenz, 46., Jg., Heft 7/1992, S. 319-323. 
Kirche als Gemeinschaft kann auch an der Qualität ihrer internen Kommunikation gemessen werden. Diese ist zugleich Voraussetzung für eine glaubwürdige Kommunikation mit der Welt."8

Aus den Dokumenten "Communio et Progressio" und "Aetatis Novae" lassen sich verschiedene Ziele für und Anforderungen an kirchliche Kommunikationsarbeit ableiten:

1. Sie hilft der Kirche in die Welt hinein zu wirken: sie informiert und orientiert über das, was Kirche ausmacht und was in der Kirche geschieht.

2. Sie will die Welt in die Kirche hinein vermitteln, indem sie aus allen gesellschaftlichen Bereichen Ereignisse und Meinungen bekannt macht.

3. Sie will unterschiedliche Standpunkte innerhalb der Kirche miteinander vermitteln, indem sie für eine offene Diskussion sorgt (die sogenannte „interne Kommunikation").

4. Sie will Informationen, Überzeugungen und Anliegen aufgreifen und auf Geschehnisse und Entwicklungen hinweisen, die andere übersehen oder unterdrücken.

5. Sie will Menschen aller Alters- und Bildungsstufen zu Kommunikation und $\mathrm{zu}$ einem kritischen, selbstverantwortlichen Umgang mit Medien befähigen.

6. Sie tritt für gerechte, dem christlichen Menschenbild verpflichtete Kommunikationsordnungen und -gesetze ein. ${ }^{9}$

Zur Erreichung dieser Ziele ist eine gute Planung kirchlicher Kommunikationsarbeit notwendig. ${ }^{10}$ Kommunikation im umfassenden Sinne meint die Gesamtheit aller einzelnen Maßnahmen, mit denen die Kirche auf verschiedenen Ebenen (Pfarrgemeinde, Dekanat, Bistum, Bischofskonferenz, Weltkirche, aber auch in Verbänden, Orden u.a.) sowohl in ihren eigenen Bereich als auch in die Welt hinein wirken kann und mit denen sie die Anfragen der Welt nach einem sinnstiftenden, erfüllten Leben aufnimmt. Im folgenden geht es jedoch vorrangig um Möglichkeiten zur Optimierung der Öffentlichkeits- und Kommunikationsarbeit. Öffentlichkeitsarbeit soll als zielgerichtete Kommunikati-

8 Zur Bedeutung der kirchlichen Medienarbeit, Appell des Bischbflichen Europäischen Komitees für Medien (CEEM), in: Communicatio Socialis, 28 (1995), Heft 4, S. 430-433. Auch abgedruckt in: multi-Media, Nr 16.17, 1995, S. 8-9.

9 Vgl. auch das Medienkonzept 90 der Evangelischen Kirche im Rheinland (1990) und die Gemeinsame Erklărung der Deutschen Bischofskonferenz und des Rates der Evangelischen Kirche in Deutschland "Chancen und Risiken der Mediengesellschaft" (= Gemeinsame Texte Nr. 10), Hannover und Bonn 1997, bes. Kap. 4.9.

10 AN, Nr. 17. 
onsarbeit verstanden werden, mit der ein "Sender"11 versucht, ein besonderes Ansehen bei einer bestimmten oder unbestimmten Zielgruppe aufzubauen bzw. eine bestehende Reputation zu pflegen und weiter auszubauen. Die Zielgruppe soll dabei zur Ansicht kommen, daß es sich beim Sender um eine vertrauenswürdige Institution handelt, die der Gesellschaft allgemein oder einer speziellen Zielgruppe im besonderen einen einzigartigen Nutzen bringt. Öffentlichkeitsarbeit kann aber auch dazu dienen, ein aus Sicht der Zielgruppe als problematisch wahrgenommenes Verhalten des Senders aufzugreifen, die Hintergründe für dieses Verhalten zu erklären und es damit als notwendig darzustellen oder auch als nicht angemessen einzugestehen und dafür eine Alternative anzubieten.

Wirkungsvolle Kommunikationsarbeit setzt das Vorhandensein von funktionierenden, transparenten Arbeitsstrukturen, von motivierten, kompetenten Mitarbeiterinnen und Mitarbeitern sowie klare und verständliche Botschaften voraus. Dazu bedarf es einerseits einer klaren funktionalen Struktur, andererseits aber auch verbindlicher Rahmenpläne für den Ablauf der Kommunikationsarbeit im Bistum. Nur ein verbindliches, umfassendes Konzept zur Kommunikationsarbeit wird den Anforderungen an die moderne Kommunikation gerecht.

Ein solchen Konzept wird in der Arbeithilfe „Struktur und Organisation kirchlicher Kommunikation" vorgestellt. Dabei geht es zunächst um die Erarbeitung eines Kommunikationsplans, dann um die Implementierung entsprechender Organisationsstrukturen und schließlich um Verfahren der Wirkungskontrolle.

Das im nachfolgenden beschriebene Modell folgt den in der Pastoralinstruktion "Aetatis Novae" dargestellten Schritten der Bestandsaufnahme (Ist-Zustand), der Bewertung und Analyse, der Planung und Definition eines Soll-Zustandes einschließlich einer Prioritätensetzung, sowie einer regelmäßigen Wirkungskontrolle. ${ }^{12}$ In dieser Form wurde es als Musterprojekt in einer Diözese erarbeitet. Abweichende Besonderheiten können in anderen Diözesen selbstverständlich Anpassungen an die jeweilige konkrete Situation erforderlich machen.

\section{Kommunikatives Handeln im Bistum}

Planvolle Kommunikationsarbeit vollzieht sich in vier Schritten, die aufeinander aufbauen:

11 Als „Sender“ wird hier der- oder diejenige bzw. eine Institution bezeichnet, der oder die ein spezielles Anliegen in den Kommunikationsprozeß einbringen möchte.

12 AN, Nr. 23-33. 
1. Aufnahme der Ist-Situation (anhand der Kriterien: Wer sind unsere Kommunikationspartner? Welche Bedürfnisse haben unsere Kommunikationspartner? Was wollen wir im Kommunikationsprozeß erreichen?)

2. Evaluierung der eigenen Stärken und Schwächen als Grundlage eigener Kommunikationsarbeit (hinsichtlich der Strukturen, der Kommunikationsmittel und -wege sowie der Themen und Inhalte) ${ }^{13}$

3. Festlegung eines Soll-Zustandes (Ziele der Kommunikationsarbeit, der damit verbundenen Zielgruppen sowie der Möglichkeiten zur Erreichung der Zielgruppen), der auch die erforderlichen Aus- und Fortbildungsmaßnahmen für die eigenen Mitarbeiterinnen und Mitarbeiter sowie sogenanne "Qualitätsstandards" enthalten muß

4. Soll-Ist-Vergleich (Wirkungskontrolle)

Koordination und fachliche Aufsicht sorgen für eine professionelle und effiziente Gestaltung der Kommunikationsarbeit und damit für eine optimale Wirksamkeit. Koordination und Kontrolle bedeuten aber nicht Zensur und inhaltliche Überwachung. (Für die Inhalte bleiben auch in Zukunft die jeweiligen Fachressorts zuständig).

Einerseits sollen die alltäglichen Kommunikationsprozesse im Bistum verbessert werden. Dazu läßt sich ein allgemeiner Medien- und Kommunikationsplan erstellen. Andererseits geht es darum, ein bestimmtes Thema wirkungsvoll in einem Bistum $\mathrm{zu}$ behandeln. $\mathrm{Zu}$ denken ist z.B. an ein Bistumsjubiläum, die Veröffentlichung eines Hirtenwortes oder einer gemeinsamen Erklärung von Deutscher $\mathrm{Bi}$ schofskonferenz und $E K D$, an ein Pastoralgespräch im Bistum, eine gemeinsame Aktion mit Jugend- oder Frauenverbänden etc. Dazu müssen besondere Maßnahmen ergriffen werden.

\subsection{Das Erstellen eines allgemeinen Kommunikationsplanes}

Ein allgemein verbindlicher Kommunikationsplan sorgt für:

- Klarheit: Das Bistum und seine nachgeordneten Einrichtungen sind eindeutig zu erkennen;

- Effektivität und Effizienz: Die vorhandenen Mitarbeiter und Mittel werden so eingesetzt, daß eine größtmögliche publizistische Wirkung erzielt wird;

- die Nutzung möglicher Synergien: Durch die Abstimmung und ein gemeinsames Vorgehen können die einzelnen Abteilungen und Einrichtungen der Diözese eine größere Wirkung erzielen als durch publizistische Alleingänge.

13 AN, Nr. 26. 
Als unabdingbare Voraussetzung für die Erarbeitung und Anwendung eines Kommunikationsplans gilt ein Gesamtverantwortlicher für die Öffentlichkeits- und Kommunikationsarbeit im Bistum (vgl. Kapitel $3)$.

Dabei sollte die Erarbeitung des allgemeinen Kommunikationsplanes unter Beteiligung aller betroffenen Einrichtungen der Diözese verlaufen. Entsprechend ihrer Einbindung kann dies von der Information und dem Vorschlagsrecht (bei nachgeordneten Dienststellen und eigenständigen Werken in der Diözese) bis hin zur aktiven Beteiligung (im Falle der Hauptabteilungen des Ordinariates) reichen.

Die Erstellung des Ablaufplanes liegt vorzugsweise bei einem Arbeitsteam, dem Vertreter der entsprechenden Hauptabteilungen und Kommunikationsfachleute angehören. ${ }^{14}$ Dieses Projektteam erfaßt alle Kommunikationsmittel und -wege, derer sich das Bistum bedienen kann und begutachtet sie im Hinblick auf ihre Wirksamkeit. Es definiert eine angemessene Sachmittelzuweisung $\mathrm{zu}$ den einzelnen publizistischen Aufgaben, sucht nach Möglichkeiten der Vereinfachung und ggf. auch der Einsparung, benennt aber auch Lücken in der bisherigen publizistischen Arbeit. Primäres Ziel ist nicht die Mitteleinsparung, sondern die Verbesserung der kommunikativen Abläufe.

Der Kommunikationsplan ist dann als vollständig anzusehen, wenn er auf folgende Fragen eine Antwort zu geben vermag:

(1) Wer sind wir? Wofür steht unser Bistum? (Selbstbild, Eigenanspruch)

(2) Was wollen wir vermitteln (Kommunikationsziel)? Mit welchem Anliegen will das Bistum in den Kommunikationsprozeß eingetreten?

(3) Wen wollen wir erreichen? (Zielgruppendefinition)

(4) Mit welchen Ressourcen (= Finanzen und Mitarbeiter) läßt sich der Kommunikationsprozeß gestalten?

Entsprechend können dann die im Bistum vorhandenen Kommunikationsmittel und -wege ausgewählt werden und gegebenenfalls Kooperationspartner oder Vernetzungen gesucht werden. Insbesondere Medienverbundprojekte (wie z.B. „Alles ALLTAG ..."15) zeigen deutlich, wie

AN, Nr. 25.

15 „Alles ALLTAG.." ist ein Medienverbundprojekt in Trăgerschaft der Landesarbeitsgemeinschaft Katholische Erwachsenenarbeit Rheinland-Pfalz (LAG), Mainz. Es wurde bisher 1992 und 1994 durchgefihrt und beinhaltete jeweils eine Fernsehserie zu christlichen Themen (1992: Dekolog; 1994: Christsein im Alltag), die bundesweit zur Fastenzeit im Dritten Programm der offentlich-rechtlichen Anstalten ausgestrahlt wurde. Dazu wurden begleitend Arbeitsmaterialien, eine Verteilzeitschrift und Gesprächskreise angeboten. 
verschiedene Kommunikationsmittel und -wege miteinander vernetzt werden können, so daß eine große Anzahl an Menschen erreicht wird, die sich zudem in einem unterschiedlich intensiven, selbstbestimmten Grad einbringen können.

Der erarbeitete Kommunikationsplan ist von der Bistumsleitung in Kraft zu setzen, um seine Verbindlichkeit deutlich zu machen.

Der allgemeine Kommunikationsplan sollte einen stetigen SollIst-Vergleich beinhalten. Dafür bieten sich insbesondere an:

- Teilnehmerzahlen

- Vergleich der eingeplanten mit den tatsächlich benötigten Finanzen

- Berichterstattung in den Medien (Anzahl, Umfang, Tendenz)

- Anfragen von Kirchenmitgliedern, Externen, Medienvertretern (Anzahl, Tendenz)

- Wirksamkeit über einen längeren Zeitraum (das Erinnerungsvermögen, auch „Recall“ genannt, und zwar gestützt und ungestützt, sowie im Zeitreihenvergleich)

- Ergebnisse von begleitenden sozialwissenschaftlichen Untersuchungen.

Die regelmäßige Erhebung solcher Daten und ein laufender Vergleich mit früheren Ergebnissen können wertvolle Hinweise zu Erfolg und Verbesserungsmöglichkeiten der getroffenen Maßnahmen geben. Eventuell kann ein Vergleich mit anderen Diözesen hilfreich sein. Wenn externe Wissenschaftler bei der Erarbeitung beteiligt sind, können sie einen wesentlichen Teil der Überprüfung übernehmen.

\subsection{Das Erstellen eines Medien- und Kommunikationsplanes für besondere Angelegenheiten und Anlässe}

Besondere Ereignisse können die Erarbeitung eines speziellen Kommunikationsplanes erfordern. Dabei ist zu unterscheiden zwischen Themen und Anlässen, die aktiv gesteuert werden können, und „Krisen-Kommunikation".

Aktiv zu steuernde Anlässe sind z.B. Diözesanfeste und -jubiläen, die Durchführung eines pastoralen Forums, die Veröffentlichung von Hirtenworten und Erklärungen oder ein Medienverbundprojekt. Je nach Inhalt, Reichweite und Zeitrahmen kann die geeignete Auswahl der Kommunikationswege und -mittel divergieren.

Demgegenüber können Störfälle aller Art die betroffenen Institutionen und Unternehmen vor unerwartete Herausforderungen stellen, da sie sich in den wenigsten Fällen vorhersehen lassen. Beispiele hierfür können eine Brandkatastrophe in einer kirchlichen Einrichtung oder die Aufdeckung eines vermeintlichen Skandals sein. Für diese Fälle ist ein 
Muster auszuarbeiten, das einige mögliche Typen derartiger Störfälle auflistet und Verhaltensmaßregeln (Was ist zu tun?) sowie Verantwortlichkeiten definiert (Wer benachrichtigt wen? Wer tibernimmt wofür die Verantwortung? Wer übermittelt wem welche Problemlösungsvorschläge?). Dies hilft, auf unerwartete Situationen zu reagieren, da Handlungssicherheit hergestellt wird, und zudem wird der jeweiligen Diözese ermöglicht, das Geschehen aktiv zu gestalten. ${ }^{16}$

\section{Die Kommunikationsstruktur im Bistum}

In einer Diözese soll die Kommunikationsarbeit neu geordnet werden. Als Ziele der Umgestaltung wurden benannt:

- Eine wirkungsvolle und glaubwürdige Beteiligung der Kirche am gesellschaftlichen Diskurs sicherzustellen

- einen verantwortungsbewußten, effizienten und zielorientierten Umgang mit den gegebenen Mitarbeitern und Mitteln zu gewährleisten

- eine hohe Professionalität der Kommunikationsarbeit zu bewirken

- systematische Medien- und Öffentlichkeitsinitiativen in Zukunft zu ermöglichen

- Synergieeffekte - wo immer möglich - zu nutzen,

- einheitliche Verfahrensregeln und Veranwortlichkeiten zu bestimmen.

Aufgrund der örtlichen Gegebenheiten wurde dies mit einer organisatorischen Umgestaltung verbunden, in deren Mittelpunkt die Schaffung einer "Hauptabteilung Kommunikationsarbeit" stand. Dieser Hauptabteilung sollten möglichst alle Funktionen zugeordnet werden, die für die Kommunikationsarbeit relevant sind.

Die Umgestaltung verlief in sieben Schritten, die aufeinander aufbauten. Sie wurden so gestaltet, daß sie den Mitarbeitern in der Medienund Kommunikationsarbeit der Diözese ein breites Feld der Beteiligung gewährten. Die nachfolgende Abbildung gibt die sieben Schritte wider:

16 Vgl. Kriebel, Wolf-Henning, Das 5-Ebenen-Modell. Konfliktkommunikation für Fuhrungskrafte, Rommerskirchen 1993. Verschiedene Unternehmen haben bereits entsprechende Plăne zur Krisenkommunikation vorbereitet (vgl. Burkhard Böndel, Markus Hennes: In der Schublade, in: Wirtschaftswoche, Nr. 12 vom 19.03.1993, S. 52-56. Christian Lipicki: Konfliktmanagement - Unkluge Politik, in: Wirtschaftswoche, Nr. 30 vom 20.07.1995, S. 58-62). Auch im kirchlichen Bereich wird hieran gearbeitet, 2.B. im Bistum Passau. 
Ablaufschema für die Einführung einer Hauptabteilung "Kommunikationsarbeit"

1. Schritt: Den EntwicklungsprozeB starten

Der Bischof gibt seinen Wunsch in der Hauptabteilungsleiterkonferenz (HALK) bekannt

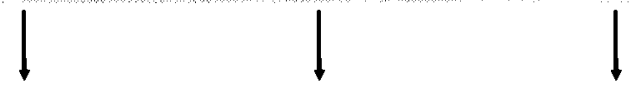

2. Schritt: Information der Mitarbeiterinnen

Aufforderung zur Beteiligung am Prozeß

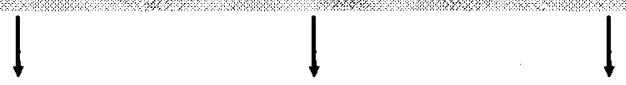

3. Schritt: Einrichten eines Projektteams

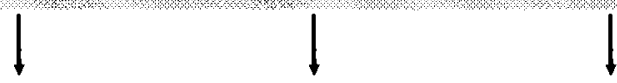

4. Schritt: Das Projektteam erarbeitet Vorschläge, außerdem sammelt und strukturiert es die Vorschláge der Mitarbeiter/innen

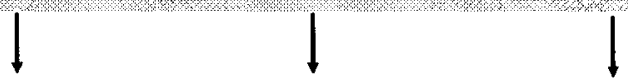

5. Schritt: Zusammenführen der Vorschläge und Erarbeiten eines Konzepts
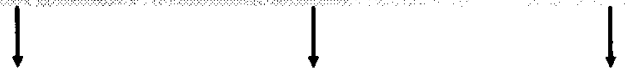

6. Schritt: Diskussion in der HALK

Verabschiedung nach Einvernehmen und Inkrattsetzung durch den Bischof

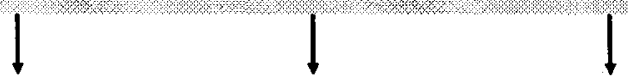

7. Schritt: Umsetzung und stete Kontrolle

Quelle: eigene Erstellung, MDG

Die Neuordnung der Kommunikationsstrukturen wurde durch die Bistumsleitung initiiert, da substantiell in bestehende Stukturen einzugreifen war. Außerdem hat der Bischof auf diese Weise - im Einklang mit den Dokumenten „Communio et Progressio“ und „Aetatis Novae"17 die Neuordnung der Kommunikationsarbeit im Bistum als Führungsauf-

17 Siehe auch CeP, Nr. 4; AN, Nr. 20. 
gabe deutlich gemacht. Dazu legte er seinen Mitarbeitern folgende Leitfragen vor:

1. Welche Kommunikationsmittel und -wege (intern wie extern) stehen uns zur Verfügung?

2. Wer ist bei uns an den jeweiligen Kommunikationspropzessen beteiligt?

3. Wie können die einzelnen Kommunikationsmittel und -wege so zusammengeführt werden, daß sich eine effiziente Struktur ergibt? Wo können sich Synergieeffekte ergeben?

4. Wo ergeben sich Stärken, die wir fördern müssen?

5. Wo zeigen sich hemmende Faktoren, die wir ausräumen müssen?

6. Wen müssen wir zusätzlich aus- und weiterbilden, um die zugewiesene Verantwortung sachgerecht wahrnehmen zu können?

7. Welche Kommunikationsmittel müssen ergänzt, gestützt, aufgebaut werden, oder welche Kommunikationsmittel sind verzichtbar?

Für die Koordination des Projektes benannte der Bischof einen Projektverantwortlichen und stattete ihn mit weitreichenden Vollmachten aus. Unter anderem wurde er als Mitglied in die Hauptabteilungsleiterkonferenz aufgenommen. Zudem wurden externe Berater hinzugezogen. Die Begleitung durch externe Moderation sollte helfen, den Zeitplan und die selbstgestellten Aufgaben einzuhalten. Zudem wurde auf diese Weise externe Fachkompetenz in neutraler Gestalt in die Arbeit eingebracht.

Die beiden zentralen Ergebnisse des dargelegten Prozesses waren:

- Die Einrichtung einer Hauptabteilung Kommunikationsarbeit, der im Beispielfall auch die Presse- und Öffentlichkeitsarbeit, die Medienarbeit (Kirchenzeitung, private Hörfunk- und Fernseharbeit, AVMedien) zugeordnet werden sollen (abweichende Verhältnisse und Traditionen in anderen Ordinariaten können andere organisatorische Ergebnisse zweckmäßig erscheinen lassen);

- Die Vereinbarung fester Regelungen zur Kommunikationsarbeit (z.B.: interne wie externe Informationen und Publikationen müssen gemeinsam mit der Hauptabteilung Kommunikationsarbeit erstellt werden, um einen gestalterisch einheitlichen und qualitativ hochwertigen Auftritt zu gewährleisten sowie Synergien auszunutzen).

Gerade der zweite Punkt ist entscheidend: Die Hauptabteilung Kommunikationsarbeit greift zwar nicht in die Inhalte der Arbeit ein (das wäre Zensur und kann nicht Sinn einer Neuordnung der Kommunikationsstrukturen sein), sie berät aber die jeweiligen Personen bzw. Institutionen hinsichtlich der optimalen Gestaltung. Des weiteren wurde festgelegt, daß die Kommunikationsarbeit regelmäßig auf ihre Effizienz überprüft wird. 
Die organisatorische Festlegung befaßt sich - bildlich gesprochen ausschließlich mit dem Gerüst. Besondere Wirksamkeit entfaltet die organisatorische und strukturelle Festlegung, wenn sie mit einem publizistischen Ablaufplan verknüpft wird, der Verfahrensweisen beinhaltet. Hilfen zur Erstellung eines solchen Kommunikationsplans wurden in Kapitel 2 vorgestellt.

\section{Wirkungskontrolle (Controlling)}

Qualifizierte, motivierte Mitarbeiter sind wesentlich für einen glaubwürdigen Dienst der Kirche. Motivation und Erfolg sind das Ergebnis einer gut geplanten Arbeit, einer sorgfältigen Personaleinsatzplanung und zielgerichteter Personalförderung (Aus- und Weiterbildung). Die der Kirche zur Verfügung gestellten Mittel (Kirchensteuer, Spenden, staatliche Zuwendungen) verlangen eine verantwortungsbewußte Verwendung, nicht zuletzt im Hinblick auf die wirtschaftliche Entwicklung.

Eine wirkungsvolle Kontrolle der ergriffenen Maßnahmen darf nicht als eine Entmündigung der Mitarbeiter begriffen werden. Kontrolle bedeutet im Idealfall, den Mitarbeitern genügend Freiraum zu überlassen, um selbstverantwortlich $\mathrm{zu}$ handeln. Kontrolle bedeutet aber auch, den Mitarbeitern regelmäßig eine Rückmeldung („Feedback“) über ihre Arbeit zu geben und ihnen bei Problemen zu helfen. Kontrolle kann auch eine Erfassung der Umfeldbedingungen mitbeinhalten, um mögliche Veränderungen frühzeitig zu erkennen und sich auf verändernde Umfeldbedingungen einzustellen. Werden diese Gesichtspunkte zusammengefaßt, spricht man von einem "Controlling-System". 18

Kontrolle in diesem Sinne beginnt mit der Erstellung eines Planes. In diesem Plan sind Kennziffern enthalten, was realistisch erreicht werden kann, wer für die Zielerreichung verantwortlich ist, welche Unterstiutzung die Verantwortlichen dazu benötigen und bis zu welchem Zeitpunkt die Ergebnisse vorliegen ("Was macht wer mit wem bis wann?"). Zudem werden vorab erkennbare Probleme definiert, die sich bereits im Planungsstadium lösen oder umgehen lassen.

Eine derartige Überwachung hilft, Erfolgsfaktoren zu erkennen, zu nutzen und zu fördern. Sie hilft aber auch, hemmende Faktoren zu bestimmen und möglichst zu vermeiden oder aber zu überarbeiten. In diesem Sinne wird Kontrolle zu Controlling, also zu einem umfassenden Steuerungssystem, das allen Beteiligten nutzt.

18 Kothes \& Klewes GmbH: Kompetenz - Kann man Öffentlichkeitsarbeit messen? Düsseldorf: Eigenverlag 1997. Dietrich Rominski: Public Relations-Erfolgskontrolle, in: Absatzwirtschaft, 48. Jg., Nr. 3/1995, S. 84-85. 
Die so gewonnenen Ergebnisse sind zielgerichtet aufzubereiten und zu dokumentieren. Sie sollten als wesentliche Informationsgrundlage den Mitarbeitern im Ordinariat zur Verfügung gestellt werden, sei es im Erfolgsfall - als Ermutigung, sei es - im Fall eines Mißerfolgs - als Hilfestellung, in Zukunft die Maßnahmen besser zu planen und durchzuführen. Insbesondere sollten diese Ergebnisse regelmäßig auf der Ebene (Erz-)Bischof, Generalvikar, Hauptabteilung „Kommunikationsarbeit" sowie in der Hauptabteilungsleiterkonferenz erörtert werden, um eine Verständigung über das weitere Vorgehen $\mathrm{zu}$ erzielen.

Alle drei Bereiche - Erarbeitung eines Kommunikationsplans für den Alltag und für besondere Anlässe, Einführung einer Hauptabteilung „Kommunikationsarbeit" und Verfahren zur Wirkungskontrolle - sind in der Arbeitshilfe "Struktur und Organisation kirchlicher Kommunikation" detailliert beschrieben und zum Teil durch Arbeitsblätter ergänzt. 
Jakob Vetsch- Das Internet als neues Medium für Thalmann die Seelsorge

"Mein Kind will wissen, ob seine Lieblingskatze in den Himmel kommt." - "Meine Freundin ist so eifersüchtig." - „Wie kann ich meiner kleinen Tochter die Dreifaltigkeit Gottes erklären?" - „Ich bin heute so deprimiert." - Fragen und Klagen an die Internet-Seelsorgerinnen und -Seelsorger. In diesem Artikel geht es darum, wie die Internet-Seelsorge (IS) entstanden ist, was sie tut und welche Ziele sie hat. Der Initiator berichtet.

\section{Entstehung}

Die IS wurde am 28. September 1995 in der Schweiz auf eine private Initiative hin - aber mit dem Segen der zuständigen kirchlichen Organe - gegründet. Dieser Dienst im Netz ist ökumenisch und wird durch ein Team betreut. Zur Zeit arbeiten acht PfarrerInnen und TheologInnen in ihrer Freizeit mit. Vertreten sind beide Konfessionen (katholisch und reformiert), beide Geschlechter (Mann und Frau), drei Länder (die Schweiz, Österreich und Deutschland), sowie die Sprachen deutsch, italienisch und englisch. Eigenständige Teams sind mittlerweile in Dänemark und Ungarn entstanden.

Wir alle - Ingenieure und Seelsorger - arbeiten freiwillig und unentgeltlich, zuweilen mit geringer Unterstützung durch die Ortskirchen. Jeder trägt größtenteils seine Kosten selbst, sodaß bisher kein Budget erstellt werden mußte. Unsre IS verfügt über ein Textangebot. Es enthält ein Wochengebet, einen Monatstext und die Jahreslosung aus der Bibel. Die User können auch Texte einsenden, die je nach Eignung veröffentlicht werden. Ferner können die User via eMail Fragen an uns richten und Probleme aufschreiben. Sie erhalten auf demselben Web innerhalb von ein bis zwei Tagen Antwort.

Die IS lagert auf vier Servern in drei Ländern: bei "Vorarlberg Online“ (VOL) in Österreich, bei "Liechtenstein Online" (LOL) im Fürstentum Liechtenstein, bei "Rheintal Online" (ROL) sowie bei „Zürich Online“ (ZHOL) in der Schweiz. Wir bevorzugen die Nähe von kommerziellen Seiten im Netz, um nahe bei den Benutzern und mitten in ihrem Alltagsleben zu sein. Im Durchschnitt erreichen uns pro Woche drei bis vier Neueingänge. Gestellt werden alle Fragen des Lebens und des Glaubens, welche heutige Menschen bewegen. Formuliert wird offen, direkt und meistens kurz. Die meisten Benutzer, die sich melden, sind 
männlich und zwischen 30 und 45 Jahren alt. Zunehmend wenden sich auch Frauen an uns.

\section{Ziel und Weg}

Als der junge Ingenieur Steffen K. G. Hefflin und ich die IS ins Leben riefen, lautete unsere Frage nicht: „Wie können wir dieses neue Medium für die Seelsorge und die Kirche nutzen?", sondern umgekehrt: „Was können wir für die vielen Leute, welche auf der Datenautobahn unterwegs sind, tun?"

Bei dieser Fragestellung wollen wir konsequent bleiben. Wir möchte eine Raststätte an der Datenautobahn sein, ein Ankerplatz für Netsurfer.

„Mein Kind will wissen, ob seine Lieblingskatze in den Himmel kommt." - „Meine Freundin ist so eifersüchtig." - „Wie kann ich meiner kleinen Tochter die Dreifaltigkeit Gottes erklären?" - „Ich bin heute so deprimiert."

Fragen und Klagen an die Internet-Seelsorgerinnen und -Seelsorger. Alltägliches, Interessantes, Belastendes. Lapidares auch, Adreßauskünfte zum Beispiel. - Fragen zu Glauben und Kirche sind am häufigsten, gefolgt von Beziehungsproblemen. - „Warum wurde im Glaubensbekenntnis der Ausdruck ,abgestiegen zur Hölle' ersetzt durch , hinabgestiegen in das Reich des Todes'?"

Seelsorge im Internet - erstaunlich, was da alles ankommt an brennenden Fragen, an Nöten und Sorgen. Dabei hat man sich vielleicht gerade noch gefragt, ob es das denn braucht, Internet-Seelsorge. Gehört die Seelsorge nicht ins persönliche Gespräch? Geht das: sich einem Computer anvertrauen?

Herr $X$ ist zwischen 30 und 40 Jahren alt. Er arbeitet mit dem Computer. Auch das Internet als modernes Informations- und Kommunikationsmittel ist ihm vertraut. Er weiß, daß es die IS gibt. Heute schweifen seine Gedanken immer wieder $a b$, er hat in seinem persönlichen Leben ein Problem zu lösen, oder er möchte ganz einfach gerne etwas wissen. $\mathrm{Zu}$ jemandem hingehen oder jemanden anrufen nein, das würde er jetzt noch nicht machen. In der Kirche ist er auch schon lange nicht mehr gewesen ... Während der Kaffeepause oder kurz nach dem Mittagessen schreibt er seine Frage in den Compi und drückt den Knopf „Senden“.

Viele elektronische Briefe erreichen die IS tagsüber. Von Leuten, die mit diesem Medium arbeiten. Die Zahl der Heimanschlüsse nimmt zu. Anfänglich eher der Männerwelt vorbehalten, entdecken es zunehmend 
Frauen. Früher ein Medium der Techniker, heute der Allgemeinheit. Anfänglich Studenten und Jüngere, surfen jetzt Menschen jeden Alters über das Datenmeer. In Deutschland und in der Schweiz gehen derzeit die Senioren ins Netz (http://www.seniorweb.de). - „Im Moment schwimme ich noch mehr als surfen", schreibt ein Achtzigjähriger. Aber es macht Spaß, eröffnet ihm neue Möglichkeiten.

Die IS ist für jene gedacht, denen dieses Medium vertraut ist. Wie die Telefonseelsorge für uns da ist, wenn wir reden wollen, ist die IS für uns da, wenn wir gerne etwas schriftlich durchgeben möchten. Eine moderne Briefseelsorge. Also nichts Außergwöhnliches. Und schon gar nicht unpersönlich. Da kommt vieles rüber an Gefühlen, an Menschlichem.

Kommt nun die Lieblingskatze jenes Kindes in den Himmel? Die Antwort lautet: $\mathrm{Ja}$, denn die ganze Schöpfung sehnt sich nach der Erlösung und wird durch Christus befreit. Auch die Katze.

Wir vom Team der IS wollen aufgrund unseres Glaubens und unserer Kenntnisse uneigennützig helfen, ohne nach Schuld zu fragen und ohne Dank zu erwarten. Darum lassen wir unseren Dienst im Netz in ständigem Kontakt zu den Benutzern kontinuierlich und ohne Druck wachsen.

Unser Ziel ist es, daß alle Benutzer des Internets dieses Angebot kennen, wie jeder Telefon-Benutzer um die Existenz der TelefonSeelsorge weiß. $\mathrm{Zu}$ diesem $Z$ weck wurde eine einfache, gut im Gedächtnis haftende Domain reserviert. Die Internet-Adresse lautet nun: „http://www.seelsorge.net“. Über die Adresse kann die IS in bisher fünf Sprachen abgerufen werden. Wir bitten die Printmedien, diese Adresse in ihre stehenden Wochenend-Hilfsdienst-Verzeichnisse aufzunehmen. Und wir bitten die Radio- und Fernsehanstalten, sie möglichst oft durchzugeben und einzublenden. Auch für alle Links im Netz danken wir aufrichtig.

\section{Kommunikation}

Die Gründung der IS hat - für uns völlig überraschend - in den Medien des deutschen Sprachraumes ein sehr großes, überwiegend positives Echo ausgelöst. Allmählich wurde uns bewußt, daß unser Dienst in der Fachwelt und in der Bevölkerung zwei Dinge ins Bewußtsein gerufen hat. Das eine betrifft das Internet, das andere die Seelsorge. Erstens: Es ist möglich, mit dem Medium des Internets qualifiziert zu kommunizieren. Zweitens: Es gibt ja noch die gute, alte Seelsorge; es gibt noch die Kirche mit ihren PfarrerInnen! Und sie ist modern. Sie kommt den Menschen entgegen. 


\section{Sicherheit}

Immer wieder gestellt wurde die Frage nach der Sicherheit der Seelsorge-eMails. Dazu ist folgendes festzuhalten: Keine Form der Kommunikation ist absolut sicher. Auch das Gespräch in der Gartenlaube kann belauscht werden. Sobald wir uns in irgendeiner Form äußern, ist es grundsätzlich möglich, daß Unbefugte Kenntnis davon erhalten. In der Seelsorge muß darauf besonders geachtet werden, denn sie ist Vertrauenssache. Die IS ist nicht sicherer (aber auch nicht weniger sicher) als die Telefon-Seelsorge oder die Brief-Seelsorge.

Angesichts der großen Datenmengen, welche im Netz transportiert werden und über die Server laufen, erachten wir es als unwahrscheinlich, daß gerade unsere eMails rausgepickt werden. Außerdem: Wer sollte ein Interesse daran haben, irgendeine Seelsorge (jene im Amtsraum, beim Telefon oder im Internet) mitzuverfolgen? Trotzdem sind Vorsicht und Behutsamkeit angebracht.

Seit dem Aufkommen des Internet-Banking wird die Frage nach der Sicherheit der Seelsorge-eMails weniger gestellt. Einem Medium, dem das Geld anvertraut wird, kann man offensichtlich auch Sorgen anvertrauen. Selbstverständlich steht unsere Arbeit strikt unter dem Seelsorge-Geheimnis. Um Mißbrauch vorzubeugen, wird in den meisten Fällen vor der Antwort Rücksprache mit dem Benutzer oder der Benutzerin genommen.

\section{Chancen}

Bei der Benutzung der IS steht eine Technik zwischen Menschen. Sie sehen und hören sich (noch) nicht. Dafür erscheint vieles ausgeblendet, was im persönlichen Gespräch störend wirken könnte: Rang, Titel, der Hauch der Amtsstube, Kleidung etc. Die Hemmschwelle ist also niedrig.

Trotzdem braucht es immer noch genug Mut, sich an unseren Dienst im Netz zu wenden. Schon nur das Eingestehen eines Problems ist oft ein weiter, mühsamer Weg. Ein 39jähriger Benutzer schrieb uns: „Trotz Internet kostet es mich eine große Überwindung, Ihnen zu schreiben ... Ich finde das echt super, was Sie hier anbieten, niemals sonst hätte ich so etwas geschrieben, geschweige denn mit jemandem darüber geredet."

„Schreiben ist ein freiheitliches Medium. Man kann das Tempo selber bestimmten, streichen, korrigieren, löschen oder absenden, und dies 24 Stunden am Tag und in der Nacht. Niemand braucht seinen Namen anzugeben. Für eine Antwort ist nur die korrekte eMail-Adresse erforderlich. Anonymităt und Distanz können für das Angehen von Problemen wohltuend sein. 
Das Internet bietet schnelle und bequeme Kontakt- und Informationsmöglichkeiten. In Windeseile werden Infos durchgegeben, und man kann auf Links und Hilfsangebote hinweisen. Schicksals- und Interessengruppen finden sich im Netz besonders schnell. Diese Erfahrungen durften wir auch mit der Gründung einer speziellen Homepage „Behinderte im Internet“ machen: „http://www.kyberna.lol.li/behinderte/welcome.htm". Für Menschen mit eingeschränkter Bewegungsfreiheit tun sich da Welten auf!

\section{Gefahren}

Ich möchte auch die Gefahren nicht verschweigen, welche dieses neue Medium mit sich bringt: die Datenflut. Der Benutzer kann seine Identität leicht verändern und das Internet als Fluchtweg vor den Erfordernissen des Alltags gebrauche.

Das Internet ist ein Bildschirm-, also ein Oberflächenmedium. Es geht weniger in die Tiefe als ein Buch oder das Gespräch.

Bei Goethe findet sich der Dialog zwischen dem König und der Schlange: Der König fragte die Schlange: „Wo kommst du her?" - „Aus den Klüften“, ersetzte die Schlange, „in denen das Gold wohnt.“ - „Was ist herrlicher als Gold?" fragte der König. "Das Licht", antwortete die Schlange. „Was ist erquicklicher als Licht?" fragte jener. „Das Gespräch“, antwortete diese.

Die IS ist eine Art elektronische Briefseelsorge. Sie erreicht nicht die Qualität eines guten Gespräches. Sie bleibt immer eine Vorform davon. Dafür ist die Hemmschwelle niedrig, und viel Gutes kann "angeklickt" werden.

Soweit wir es überblicken können, schafft das Internet kaum neue Probleme, aber es akzentuiert bestehende. Gerade deshalb sind wir davon überzeugt, daß es die Seelsorge im Netz in allen Sprachen und Ländern braucht!

(Homepage: http://www.seelsorge.net/javetha) 
Die Verteilzeitung "Dialog" an alle katholischen Haushalte der Diözese (Auflage 720.000), das Magazin für „alle, die für Kirche unterwegs sind - _thema_kirche_" (Auflage 26.000), ein Relaunch der „Wiener Kirchenzeitung" (Auflage 31.000) und eine Lizenz für ein eigenes Radio („Radio Stephansdom") sind die äußeren Merkmale einer völlig reformierten Öffentlichkeitsarbeit in der Erzdiözese Wien. Eine wichtige Voraussetzung dafür war aber zunächst eine strukturelle Veränderung und ein Denkmusterwechsel betreffend der Kommunikationsarbeit. Kommunikation muß in einem Unternehmen und damit auch in der kirchlichen Struktur zunächst als Chefsache, damit als ureigenste Aufgabe des Bischofs verstanden werden. Dem müssen die Hilfsinstrumente und Strukturen entsprechen. Die Öffentlichkeitsarbeit wurde daher als "Amt der diözesanen Kurie" etabliert. Der Kommunikationsdirektor ist damit im Organigramm im selben Rang wie der Finanzkammerdirektor oder Pastoralamtsleiter - mit Sitz im Konsistorium, dem formell engsten Beraterkreis des Erzbischofs nach dem Bischofsrat.

Der Kommunikationsdirektor ist zudem Vertreter der Erzdiözese im Eigentümergremium der Wiener Domverlags-GmbH. Das ist jener Wirtschaftsbetrieb, der neben Verlag und Buchhandlungen auch Medieninhaber der diözesanen Printprodukte (Dialog, _thema_kirche_, Wiener Kirchenzeitung) ist.

Die operative Arbeit wurde nach Zielgruppen entwickelt. Wesentlich dabei ist, daß auch die interne Kommunikation als geplante und organisierte Arbeit verstanden wird. Dafür steht etwa ein Schnellfaxsystem, mit dem rund Hundert der wichtigsten Repräsentanten der Diözese (vom Erzbischof bis zur Dechantenebene) erreicht werden. Diese erhalten Informationen über "heiße Themen" im voraus. Und das Monatsmagazin _thema_kirche _, mit dem etwa 26.000 Mitarbeiterinnen und Mitarbeiter (haupt- und ehrenamtlich) Informationen aus erster Hand erhalten.

Integrierter Bestandteil dieses Monatsmagazins ist auch das amtliche Diözesanblatt, in dem die kirchenrechtlich nötigen Verlautbarungen veröffentlicht werden. _thema_kirche_ hat seither eine Grundversorgung an interner Information aufgebaut. Ein leitender Mitarbeiter der mittleren Führungsebene formuliert einmal: „Bis _thema_kirche_erschienen ist, war ich auf Gerüchte angewiesen."

Darüber hinaus werden all jene in den Blick genommen, die sich zur 
katholischen Kirche zugehörig fühlen. Über $80 \%$ von ihnen haben nur einen sehr losen pfarrlichen Kontakt. Hier ist es Aufgabe der Kirche, offen auf diese Menschen zuzugehen und nicht bloß zu warten, bis sie sich auf den Weg machen (Bringschuld). Nur das offene Zugehen entspricht dem missionarischen Grundauftrag der Kirche. Das Monatsmagazin „Dialog“ ist ein medialer Versuch einer ersten Kontaktaufnahme, zum Preis von 1\% des Diözesanbudgets.

Dialog wurde unter Begleitung von Marktforschung entwickelt und ein Jahr lang im Probebetrieb getestet (vgl. Communicatio Socialis 3/97). In dieser Zeit konnte "Dialog" die Zustimmungswerte, die schon bei der Null-Nummer solide hoch waren, weiter steigern. So bewerten $79 \%$ der Katholiken die Idee positiv, Dialog an alle katholischen Haushalte $\mathrm{zu}$ verschicken. Und auch der Kosteneinsatz aus Kirchenbeiträgen findet eine Zustimmung weit jenseits der Zweidrittelgrenze, nämlich bei 73\% (Quelle Gallup). Die „erwünschten Nebenwirkungen“ stellen sich bereits ein: Plötzlich sind kirchliche Angebote, die entsprechend beworben werden, ausgebucht. Und der Leserdialog begann: in Hunderten Leserbriefen von Menschen, die bisher nur einen losen kirchlichen Kontakt hatten. Daß es sich nicht um ein PR-Blättchen, sondern um ein journalistisches Produkt handelt, zeigt auch der journalistische Erfolg: Exklusive Berichterstattung des Dialogs wird von anderen Medien zitiert, bis hin zur Hauptnachrichtensendung im Fernsehen.

Wer durch die Etablierung eines Gratismediums den zwangsläufigen Tod der Kirchenzeitungen (in Deutschland „Bistumsblätter“) prognostizierte, wie etwa Michael Schmolke (vgl. Communicatio Socialis 3/97), der irite gewaltig. Tatsächlich ist keine signifikante Auswirkung auf das Kaufverhalten ablesbar. Allerdings ist der jährliche Auflagenverlust von rund 5\% in der Erzdiözese bislang ungebrochen. Dem gilt es entgegen zu steuern. Da ein Medium wie Dialog, das alle Katholiken erreicht, zwangsläufig für jene, die einen intensiveren Kontakt zur Kirche haben, oftmals als zu leichtgewichtig empfunden wird, ist die Marktchance für eine Kaufzeitung bei den regelmäßigen Gottesdienstbesuchern ungebrochen. Für dieses Intensivsegment wird derzeit die Wiener Kirchenzeitung neu positioniert.

Sie kann sich nun von der Last befreien, den unmöglichen Spagat von der Insiderinformation für Mitarbeiterinnen und Mitarbeiter bis zur Involvierung der sogenannten Fernstehenden zu schaffen.

Für diesen Umgestaltungsprozeß (thematisch, grafisch, vertrieblich) wurde die Deutsche Medien-Dienstleistung-Gesellschaft (MDG) zur Beratung und Begleitung angeheuert.

Auf völlig neues Terrain im deutschen Sprachraum begibt sich die 
Erzdiözese Wien mit dem Antrag für eine eigene Radiolizenz. Den Zuschlag hat sie bereits erhalten. Als Sendestart ist der September 1998 angestrebt.

Ein eigenes Radioengagement der Kirche wurde im Vorfeld kontrovers diskutiert. Versteht man die Kirche aber als Sendeunternehmen, das den missionarischen Grundauftrag hat, die Frohe Botschaft unter die Menschen zu bringen, gilt eigentlich eine Art Beweis-Umkehr: Es gilt nicht $\mathrm{zu}$ begründen, warum man sich des elektronischen Mediums bedienen möchte, vielmehr gelte es umgekehrt zu belegen, warum dieser Weg als völlig untauglich gelten soll. Daher ist es nicht plausibel, daß die Kirche, die sich selbstverständlich der Printmedien bedient, auf dieses elektronische Medium von vornherein verzichten soll. In Österreich stellt sich zudem die günstige Startvoraussetzung, daß die Kirche die Lizenz bereits zu Beginn der Entwicklung des Privatradiomarktes angestrebt und erhalten hat - nicht erst $z \mathbf{u}$ einem sehr späten Zeitpunkt, wie zum Beispiel die Evangelische Kirche für ihr Projekt "Radio Paradiso" in Berlin.

Mit der Radiolizenz soll in der Gesellschaft das Segment der Sinnsucher - unabhängig vom Grad ihrer kirchlichen Bindung - angesprochen werden.

Rechtlich und wirtschaftlich ist das Radioprojekt als eine kirchliche Stiftung organisiert, dem gemäß dem österreichischen Konkordat auch Öffentlichkeitsrecht zukommt. Die Geschäftsführung wird derzeit von Kommunikationsdirektor in Personalunion wahrgenommen.

Über diese kirchlichen Zielgruppen hinaus, gilt es die Gesellschaft insgesamt durch eine offensive Medienarbeit zu erreichen. Dafür wurde in der Erzdiözese die Pressestelle ausgebaut - auch technisch durch seinen Anschluß an die Austria Presse Agentur (apa), der gewährleistet, daß die Kirche gesellschaftliche Entwicklungen nicht erst aus der Tagespresse erfährt und umgekehrt die diözesanen Pressemitteilungen zeitgleich auf allen Bildschirmen der profanen Redaktionen flimmern. Aktive Vermittlung von Interviewpartnern sowie das Coaching der kirchlichen Vertreter von Medienauftritten gehört hier zur Tagesarbeit.

Der vielfach geäußerte Verdacht, die Konzentration auf eigene Medien könnte oder würde einen Rückzug aus der profanen Medienlandschaft bedeuten, ist in der Praxis widerlegt. Auch unter Ausklammerung der kirchlichen Präsenz im Rahmen der aktuellen Turbulenzen der Kirche (Causa Kardinal Groer) ist die mediale Präsenz der Kirchenleitung deutlich höher als in früheren Jahren.

All diese Maßnahmen wurden in einem Zeitraum von zwei Jahren durchgeführt ("_thema_kirche_" kam bereits nach 3 Monaten auf den 
Markt, "Dialog" nach 11 Monaten, der Startschuß für die „Kirchenzeitung Neu" und den Radiosendebetrieb fallen ins Jahr 3 des Bestehens des Amtes für Öffentlichkeitsarbeit der Erzdiözese Wien). 


\section{Stefan Hartwig Mutiert Werbung zur Unterhaltung?}

Vergangene medienkritische Diskussionen um die Unterschwelligkeit der Werbung haben ein Phänomen außer Acht gelassen: Werbung wird zunehmend mit Unterhaltsabsicht betrachtet und ihr Unterhaltungscharakter steht scheinbar zunehmend im Vordergrund.

Die Cannes-Rolle, ein Zusammenschnitt von Spots aus dem jährlichen Werbefestival, hat inzwischen Kultstatus erreicht und die avantgardistischen Kinos und das Fachpublikum verlassen und sich in Main Stream Kinos und Videotheken ausgebreitet. Versandfirmen und Videotheken vertreiben Filme wie „Rendezvous unterm Nierentisch. Die Wirtschaftswunderrolle“, „American TV Commercial Classics“, „Oh, wie süß! Die Süßwarenrolle“, „Top of the best. Highlights aus 5 Jahren Cannes-Rolle". Die Liste ließe sich beliebig erweitern.

Die reine Unterhaltungsabsicht bei den teilweise längst nicht mehr aktuellen Spots erstaunt so wie die Vielfalt des Angebots. Es stellt sich natürlich die Frage, ob der Zuschauer solcher Videos auch aktuelle Werbung unkritisch betrachtet, weil er sie nicht anders als einen Spielfilm bewertet.

Das Fernsehen hat Schritt gehalten. Das Hauptwerbemedium behauptet auch in dieser Hinsicht seinen Platz. Der Sender Sat.1 strahlte die Serie "Die witzigsten Werbespots der Welt" aus. Eine Fernsehzeitschrift kommentiert eine Folge entsprechend enthusiastisch: „von dänischer Cola-Werbung bis zum chilenischen Strumpthosen-Spot. Fritz Egner präsentiert die besten Werbefilme weltweit, darunter der diesjährige Festivalsieger von Cannes".

RTL 2 zog nach und sendete "Hotzpotz“. Wie die bewußte Verballhornung des Begriffes "Hot Spots" andeutet, handelt es sich um eine Vorstellung und eine Art Charts gegenwärtiger Spots. Zusätzlich werden Produktion, Special Effects, Darsteller und Regisseure vorgestellt. Verschiede Publikums- und Programmzeitschriften folgen diesem Trend eines eigenen Werbe-Rankings. Dadurch werden sie auch für Werbekunden interessanter, die eine Einschätzung ihrer Anzeigen gleich kostenlos der nächsten Ausgabe entnehmen können.

Neben der Entdeckung des Unterhaltungscharakters von Spots und Anzeigen ist auch ein vermehrtes, meist populärwissenschaftliches Interesse an Werbung zu entdecken. Das betrifft sowohl Ausstellungen in Museen, als auch zahlreiche Veröffentlichungen auf dem Buchmarkt. So konnte man nicht nur in Hamburg die Ausstellung „Vom SandwichMan zur Cannes-Rolle. Die Kunst zu werben" besuchen und selbst nach 
Meinung der konservativen Zeitung "Die Welt" erfahren, „wie die Werbung unser Leben verändert hat". In Wien war die Ausstellung des Werbesujets eines einzigen Produktes zu sehen, der Wodka-Marke Absolut. Nach Auffassung der Aussteller war es eine Weltpremiere, denn „erstmals werden im Museum für angewandte Kunst Originale jener zeitgenössischen Kunstsammlung ausgestellt, die der legendären Werbekampagne von Absolut Vodka zugnundeliegt. Diese hat Geschichte gemacht - als Kommunikationstrategie und als Kooperation von Wirtschaft und Kunst".

Die Premiere war eigentlich anderer Art, es wurde gar nicht versucht, die Vermischung von Werbung, Öffentlichkeitsarbeit, Mode und Kunst zu negieren. Im Gegenteil - es zerbrechen Grenzen zwischen "fashion, Public Relations and marketing long before the first drink is poured". Deutlicher kann sich die soziale Akzeptanz von Werbung gar nicht zeigen.

Der Wodka-Hersteller lud unabhängig von dieser Ausstellung in einem Wiener Café "zur Vorführung und Verführung“ ein. Die Kampagne und ihre Sujets wurden gezeigt und man konnte an einer Verlosung teilnehmen und Musik und Drinks zur Werbung konsumieren. Oder war es umgekehrt?

Werbung ist offenbar interessant genug, um für sich allein Unterhaltungswert zu haben und das Produkt allenfalls als Beiwerk zu beachten. Schließlich kann man sich auch das Buch mit Fotografien von Bildern, Glas-Design und Skulpturen so renommierter Künstler wie Keith Hearing, Andy Warhol und Kenny Scharf kaufen. Der Umweg über eine mehr als wenig kritiklose Kommentierung dieser Art von Werbung entfällt damit.

Und was sind schließlich Videoclips in Sendern wie VIVA, VIVA 2 oder MTV, die österreichweit per Kabel oder Satellit empfangbar sind, anderes als Werbespots für die CDs und Konzerte der Interpreten? Der längst nicht mehr stigmatisierende Hinweis "Werbung" wird allerdings nicht eingeblendet. Entscheidet er über Art des Programminhaltes?

Berichterstattung über Werbung hat sich auch auf Printmedien ausgedehnt. Während "Die Welt" das Thema gleich in einer eigenen Rubrik im Wirtschaftsteil behandelt, weil „Themen aus der schillernden Welt der Werbung großes Interesse finden", berichten österreichische Tageszeitungen noch eher verschämt in Rubriken mit Titel wie „Medien“ oder gleich auf der Wirtschaftsseite in unverdächtigerer Weise über Werbemaßnahmen. Weniger berichten sie über Untersuchungen soziokultureller Werbefolgen.

Auch der „Stern“, "Die Woche“, „Der Spiegel“ oder „Focus" berichten regelmäßig über neue und oft besonders drastische Werbemetho- 
den, sei es daß man in Berlin mit Werbeeinschaltungen kostenlos telefonieren kann oder Jeanshosen an Politiker verschickt werden, damit diese sich "gut fühlen“.

Der Teil der Berichterstattung, der im Prinzip nichts anderes tut als eine bestimmte Kampagne oder einzelne Maßnahme (und damit auch Marke) vorzustellen, ist das kostenlose Ergebnis geschickter Öffentlichkeitsarbeit.

Neben dem Merchandising der Rundfunksender (ein ORF-Shop ist „immer in unserer Nähe") wird auch in großem Umfang Musik aus der Werbung vertrieben: "Stay a little Bit longer" (der Hit aus dem Bitburger TV-Spot von "Virgin Records"), „Let's work together" (mit dem Song aus der Aral-Werbung von „Columbia“) oder „Die besten Songs aus der Werbung. Volume 1-8" (von Edel, Gesellschaft für Produktmarketing und RTL).

Der Rezipient kann also - verallgemeinert gesagt - das Produkt benutzen, sowohl zur Unterhaltung als auch ungefragt Spots und Musik aus der Werbung rezipieren und an Werbe-Rankings teilnehmen. Dieses Szenario stellt zwar einen extremen und konstruierten Fall dar, zeigt aber die überraschenden Möglichkeiten der Konfrontation mit Werbung im Alltag. Kommunikationswissenschaftler identifizieren in unseren Mediengesellschaften zunehmend das Phänomen der Hybridisierung, die Vermischung und Vernetzung verschiedenster Medientypen und Medieninhalte. Unterscheidungsmerkmale verschwinden und Kategorien verschwimmen. Aus Information, Entertainment und Werbung wird ein Konvolut, dessen Name ,infotainment“ über den drohenden Orientierungsverlust hinwegtäuscht.

Nach Auffassung von Sigrid Löffler ist auch die Gestaltung von Medieninhalten inzwischen von der Werbung evoziert. Das offenbart sich besonders bei Neugründen von Printmedien wie "News" und „Focus“, deren Werbeschaltungen ihre älteren Konkurrenten „Profil“ und "Der Spiegel" inzwischen weit übertreffen. Diese Printmedien übernehmen wie eine Art "gedrucktes Fernsehen" oder "Zeitschriften für Zapper und Switcher".

Hybridisierung bedeutet den Verlust von Kategorien wie Ernsthaftigkeit oder Glaubwürdigkeit, wie Irmela Schneider befürchtet.

Währenddessen ergänzen Siegfried J. Schmidt und Brigitte Spieß, daß der Verlust von Orientierungswissen durch den Verlust solcher Kategorien auch den Verlust von Konventionen mit sich bringt. Durchbrechung der Konvention bedeutet dabei auch Verwischung der gesetzlich mehr oder weniger genau definierten und sanktionierten Trennlinien zwischen Information, Unterhaltung und Werbung. Dazu kommen neue Medien wie das Internet, die von der Werbung kommer- 
zialisiert werden, ohne daß die Gesetzgebung Schritt halten kann. Die Werbeaufwendungen im Internet haben sich 1997 um rund 400 Prozent auf 24 Millionen DM (168 Millionen ÖS) erhöht. Für 1998 rechnet man bereits mit 50 Millionen DM.

Möglicherweise ist schließlich im Zuge der Vermischung von Werbung, Unterhaltung und Information Glaubwürdigkeit alleine für den Rezipienten gar kein Auswahlkriterium für den Medienkonsum mehr. Das Rezeptionsinteresse kann auch selbst hybridisiert werden und bei allen Medieninhalten sowohl aus Unterhaltungsabsicht als auch aus Informationsbedürfnis gleichzeitig bestehen.

Dieser Entwicklung sollte mehr mediale Aufmerksamkeit entgegengebracht werden, als es die Ausstrahlung des Cannes-Rolle oder der "witzigsten Werbespots der Welt" vermögen.

S. Löffler, Die neuen Medien, in: S. Löffler, Kritiken, Portraits, Glossen, Wien 1995

S. J. Schmidt /B. Spieß, Die Kommerzialisierung der Kommunikation. Fernsehwerbung und sozialer Wandel 1956-1989, Frankfurt/M. 1997

I. Schneider, Hybridkultur. Eine Spurensuche, in: C. W. Thomson (Hg.), Hybridkultur. Bildschirmmedien und Evolutionsformen der Künste, Siegen 1994 


\section{Resolution on computer networking}

Issued by the Committee on Communications of the United States Catholic Conference

Pope John Paul II has called the mass media one of the "modern equivalents of the Areopagus" of Athens, one of "the new sectors in which the Gospel must be proclaimed" (cf. "Redemptoris Missio", \# 37c). A relatively new aspect of the media which is already re-shaping the ways in which we interact with one another is computer networking and, in particular, the Internet. The Committee on Communications of the United States Catholic Conference wishes, by this resolution, to affirm the value of these new means. We also wish to identify some concerns about their potential misuse which can divert them away from service to the truth and to the common good.

With regard to their use within the Church community, many dioceses, parishes, religious communities, schools, and other Church institutions and organizations are already effectively employing these new means. An outstanding example is the Holy See's establishment of its Web site. By the convenient and almost instantaneous communications they make possible, these new means help bring about an enhanced sense of working together, whether in a pastoral center, a diocese, or a far-flung religious community. This conquest of time and space offers new ways to gather people together for a common purpose. We encourage an extensive exchange of information and ideas among Church leaders and communicators about creative uses of computer networking and the Internet for service to the Church's pastoral mission.

The Church and her institutions (including schools, social service agencies, and health care facilities) and all persons regardless of income should have access to these means of communications. As computer networks become more essential to functioning within society, legislation and regulation regarding them should guarantee that access.

At the same time, we wish to raise up our concerns about the possible abuses of computer networking.

Our principal concern is that what is presented on the Internet and elsewhere as "Catholic" be authentically so and that truly Catholic sites not be linked to sites which contradict Church teaching und practice. Among the preventive steps which ought to be considered are:

- a review of canon law to determine whether the canons which govern printed matter and the authorization of the use of the name "Catholic" can be applied by diocesan Bishops in this regard; 
- development by local dioceses of criteria for establishing Web sites and for linking Web sites to one another and the designation of appropriate diocesan offices to oversee their application;

- development, maintenance, and promotion by the Conference, in consultation with local dioceses, of a list of reliably Catholic sites on the Internet.

Related concerns are:

- that sites which do not accurately reflect Church doctrine or devotion not be presented in existing official or semi-official guides as "Catholic"sites;

- that the integrity of Church documents not be compromised;

- that the Church community and the general public not be deceived by individuals who use the means to misrepresent their relation to the Church or to use the name "Catholic" to exploit extremely vulnerable people and lure them into deceptive and even fraudulent schemes which drain their resources.

These new means of communications can also make an important contribution to the common good of society. Their use should enhance the unity of the human family rather than increase its divisions.

Thus we urge that the capacity of these new means for the nearly instantaneous dispersal of information world-wide be employed whith a commitment to the truth and to the common good.

We also wish to express our profound concern about the immoral uses to which these networks are being put in ways that harm adults and children both, denying them their human dignity and turning them into objects of exploitation. The fact that children are often more "computer literate" than their parents or guardians can place them at special risk.

As these means become necessities for earning one's income or simply going about the activities of daily living, we pledge our support for the poor and vulnerable having access to them.

Computer networking is a rapidly changing reality. This articulation of concerns should not be construed to be exhaustive or final. In order to affirm the importance these means of communications and to raise up our concerns in a timely fashion, we limit ourselves at present to this brief resolution.

Approved September 10, 1997, published in USCC News

September 16, 1997 


\section{„In der Kraft des Heiligen Geistes die Hoffnung vermitteln"}

Botschaft Papst Johannes Paul II. zum 32. Welttag der sozialen Kommunikationsmittel 1998

Liebe Schwestern und Brüder!

1. In diesem zweiten von drei Jahren, die zum Großen Jubiläum des Jahres 2000 hinführen, richten wir unsere Aufmerksamkeit auf den Heiligen Geist und sein Wirken in der Kirche, in unserem Leben und in der Welt. Der Geist ist der „Hüter der Hoffnung im Herzen des Menschen" (Dominum et vivificantem, 67). Aus diesem Grund folglich lautet das Thema für den 32. Welttag der Kommunikationsmittel: "In der Kraft des Heiligen Geistes die Hoffnung vermitteln."

Die Hoffnung, in der der Geist die Gläubigen trägt, ist vor allem eschatologischer Natur. Sie ist Hoffnung auf das Heil - Hoffnung auf den Himmel, Hoffnung auf die vollkommene Gemeinschaft mit Gott. In solcher Hoffnung haben wir, wie es der Brief an die Hebräer ausdrückt, „einen sicheren und festen Anker der Seele, der hineinreicht in das Innere hinter dem Vorhang; dorthin ist Jesus für uns als unser Vorläufer hineingegangen" (Hebr 6,19-20).

2. Die eschatologische Hoffnung, die in den Herzen der Christen lebt, hat tief innerlich Bezug zur Suche nach Glück und Erfüllung in diesem Leben. Hoffnung auf den Himmel erzeugt echte Sorge um das Wohlergehen der Menschen hier und jetzt. "Wenn jemand sagt: Ich liebe Gott!, aber seinen Bruder haßt, ist er ein Lügner; denn wer seinen Bruder nicht liebt, den er sieht, kann Gott nicht lieben, den er nicht sieht" (1 Joh 4,20). Erlösung, die die Heilung der Gott-Mensch-Beziehung durch Gott bedeutet, geht einher mit der Heilung unserer Beziehungen untereinander, und die aus der Erlösung stammende Hoffnung zielt auf diese doppelte Heilung. Deshalb ist es so wichtig, daß Christen sich auf das Große Jubiläum am Beginn des Dritten Jahrtausends vorbereiten, indem sie ihre Hoffnung auf das endzeitliche Kommen des Gottesreiches erneuern und auch scharfsichtiger die Zeichen der Hoffnung lesen, die sie in der sie umgebeneden Welt finden. Unter den Zeichen der Hoffnung sind folgende: wissenschaftlicher, technologischer und besonders medizinischer Fortschritt im Dienst am menschlichen Leben; eine größere Bewußtheit unserer Verantwortung für die Umwelt; Bemulhungen, Friede und Gerechtigkeit wiederherzustellen, wo sie verletzt worden sind; ein Verlangen nach Versöhnung und Solidarität unter den Völkern, besonders in der komplexen Beziehung 
zwischen dem Norden und dem Süden der Welt. Auch in der Kirche gibt es viele Zeichen der Hoffnung, darunter ein aufmerksameres Hinhören auf die Stimme des Heiligen Geistes und seine Eingebung, Charismen zu akzeptieren und die Laien zu fördern; eine tiefere Verpflichtung zur Einheit der Christen und eine wachsende Anerkennung der Bedeutung des Dialogs mit anderen Religionen und mit der zeitgenössischen Kultur (vgl. Tertio Millennio adveniente, 46).

3. Christliche Medienschaffende werden Hoffnung glaubwürdig vermitteln, wenn sie zunächst Hoffnung in ihrem eigenen Leben erfahren, und dies wird nur geschehen, wenn Sie Mănner und Frauen des Gebetes sind.

In der Kraft des Heiligen Geistes befähigt uns das Gebet, „stets bereit (zu sein), jedem Rede und Anwort zu stehen, der nach der Hoffnung fragt, die man in uns sieht" (1 Petr 3,15$)$. So lernt der in den Medien tätige Christ, die Botschaft der Hoffnung den Männern und Frauen unserer Zeit mit der Kraft der Wahrheit darzustellen.

4. Man darf niemals vergessen, daß mediale Kommunikation nicht ein utilitaristisches Tun ist, einfach darauf gerichtet, zu motivieren, zu überreden oder zu verkaufen. Noch weniger ist sie ein Vermittler für Ideologie. Die Medien können gelegentlich die Menschen auf Konsumeinheiten oder konkurrierende Interessengruppen reduzieren oder Zuschauer, Leser und Hörer als bloße Zahlen manipulieren, von denen man sich einen Vorteil verspricht - ob Verkauf von Produkten oder politische Unterstützung; all das zerstört die Gemeinschaft. Es ist die Aufgabe von Kommunikation, Menschen zusammenzubringen sowie ihr Leben zu bereichern, und nicht, sie $z u$ isolieren und auszubeuten. Die Mittel der sozialen Kommunikation können - richtig genutzt - dazu beitragen, eine menschliche Gemeinschaft zu schaffen und aufrechtzuerhalten, die auf Gerechtigkeit und Liebe beruht; und insoweit sie das tun, werden sie Zeichen der Hoffnung sein.

5. Die Medien der gesellschaftlichen Kommunikation sind in der Tat der neue "Areopag" der Welt von heute - ein großes Forum, das im besten Fall den Austausch wahrheitsgemäßer Informationen, konstruktiver Ideen und echter Werte ermöglicht und so Gemeinschaft schafft. Dies ist dann wieder für die Kirche eine Herausforderung, in ihrem Kommunikationsansatz die Medien nicht nur zu Verbreitung des Evangeliums zu gebrauchen, sondern tatsächlich sogar zur Integration der Botschaft des Evangeliums in die von modernen Kommunikationsformen geschaffene "neue Kultur" mit ihrer „neuen Sprache, mit neuen Techniken und mit einer neuen psychologischen Haltung" (Redemptoris missio, 37).

Christliche Medienschaffende brauchen eine Ausbildung, die sie 
befähigt, wirkungsvoll in einer Medien-Umgebung dieser Art zu arbeiten. Eine derartige Ausbildung wird einen breiten Themenkreis umfassen müssen: Unterweisung in technischen Fähigkeiten, in Ethik und Moral, menschlicher Kultur, Philosophie, Geschichte, Sozialwissenschaften und Ästhetik. Vor allem anderen jedoch muß diese Ausbildung eine Formung im inneren Leben sein, im Leben des Geistes. Medienschaffende, die Christen sind, sollten Männer und Frauen des Gebets sein, das vom Geist erfüllt ist, und so immer tiefer in Gemeinschaft mit Gott eintreten, um in ihrer Fähigkeit zu wachsen, Gemeinschaft unter ihren Mitmenschen fördern $z \mathfrak{u}$ können. Sie müssen in Hoffnung "geschult" werden durch den Heiligen Geist, "die Hauptkraft der Neuevangelisierung" (Tertio Millennio adveniente, 45), so daß sie anderen Hoffnung vermitteln können.

Die Jungfrau Maria ist das vollendete Vorbild der Hoffnung, die christliche Medienschaffende in sich selbst $\mathrm{zu}$ entfachen und mit anderen zu teilen suchen. „Maria bringt die Sehnsucht der Armen Jahwes voll zum Ausdruck und leuchtet als Vorbild für alle, die sich mit ganzem Herzen den Verheißungen Gottes anvertrauen" (Tertio Millennio adveniente, 48). Da die Kirche ihren Pilgerweg zum Großen Jubiläum geht, wenden wir uns an Maria, deren tiefes Hinhören auf den Heiligen Geist die Welt für das große Ereignis der Menschwerdung, die Quelle aller Hoffnung, geöffnet hat.

Aus dem Vatikan, am 24. Januar 1998,

Fest des hl. Franz von Sales.

(aus: L'Osservatore Romano Deutsch vom 06.02.1998) 
Johannes Film als Träger und Vermittler von Paul II. Bildung und Kultur

Ansprache beim Internationalen Studientreffen

am 1. Dezember 1997 in Rom

Herr Kardinal,

meine Damen und Herren!

1. Es ist mir eine Freude, Ihnen, die Sie an dem Internationalen Studientreffen: „Film, Vermittler von Spiritualität und Kultur" teilnehmen, zu begegnen. Dem Päpstlichen Rat für die Kultur und dem Päpstlichen Rat für die sozialen Kommunikationsmittel, die in Zusammenarbeit mit der "Rivista del Cinematografo dell'Ente dello Spettacolo" dieses Treffen veranstaltet haben, bringe ich meine Wertschätzung zum Ausdruck.

Zusammen mit meinem herzlichen Grußwort an Sie möchte ich auch diejenigen grüßen, die im Bereich von Kultur, Kommunikation und Film mit Thnen zusammenarbeiten, und Ihnen allen eine zunehmend erfolgreiche Arbeit wünschen.

2. Vor kurzem hat der Film das erste Jahrhundert seines Bestehens vollendet, und er zieht weiterhin das Interesse der Öffentlichkeit an, die ihn als Gelegenheit, ein Schauspiel zu erleben, wahrnimmt. Der Film besitzt aber auch die Fähigkeit, das persönliche Wachstum zu fördern, wenn er den Menschen zu ästhetischer und geistiger Erhebung führt. Aus diesem Grund möchte die Kirche im Kontext dieses ersten Festivals „Tertio Millennio“ ihren Beitrag leisten zur Reflexion über die geistigen und kulturellen Werte, deren Träger der Film sein kann.

Die Kirche hat die Bedeutung der sozialen Kommunikationsmittel schon seit deren Aufkommen anerkannt als nützliche Instrumente zur Bekanntmachung und Wertschätzung der menschlichen und religiösen Werte, die das Reifen der Person unterstiitzen. Sie hat die auf diesem schwierigen Gebiet Arbeitenden auf ein hohes Verantwortungsbewußssein hingewiesen. Der Film steht Seite an Seite mit diesen Mitteln und bedient sich dabei seiner eigenen Sprache, die es gestattet, Personen verschiedener Kulturen zu erreichen. In den ersten hundert Jahren seines Bestehens ging der Film Seite an Seite mit anderen Kunstformen, die schon vor ihm bestanden hatten, und verband sich mit ihnen auf neue und eigene Weise, und so brachte er Meisterwerke zustande, die jetzt ein wesentlicher Teil des gemeinsamen kulturellen Erbes sind. Es war ein Fortschritt auf technischer wie auf künstlerischer und menschlicher Ebene. Im ersten Jahrhundert des Films sind bedeutende Fortschritte zustandegekommen, die ihm große Ausdrucksmöglich- 
keiten geboten haben, wenn die Technik es auch in manchen Fällen mehr auf die besondere Wirkung als auf den Inhalt abgesehen hat.

3. Der wirkliche Fortschritt dieser modernen Form der Kommunikation bemißt sich nach ihrer Fähigkeit, Gehaltvolles zu übertragen und Lebensmodelle vorzustellen. Wer zu einer der in verschiedenen Formen angebotenen Filmvorführungen geht, spürt die Kraft, die davon ausgeht, denn der Film vermag das Denken und Verhalten von ganzen Generationen zu lenken. Darum ist es von Bedeutung, daß er positive Werte anzubieten hat und die Würde der menschlichen Person respektiert.

Neben Filmstreifen, die mehr den Charakter zur Unterhaltung aufweisen, gibt es eine Menge von Filmen, die mehr zugänglich sind für existentielle Probleme. Deren Erfolg ist vielleicht weniger lautstark, aber sie spiegeln die Arbeit großer Meister wider, die durch ihr Werk dazu beigetragen haben, das kulturelle und künstlerische Erbe der Menschheit zu bereichern. Angesichts solcher Filme wird der Zuschauer zum Nachdenken über Aspekte einer ihm manchmal unbekannten Wirklichkeit gebracht, die Bilder geben ihm innerlich Fragen auf, und er spiegelt sich in ihnen, er konfrontiert sich mit verschiedenen Perspektiven und kann nicht gefühllos bleiben für die Botschaft, die ihm das Filmwerk übermittelt.

Der Film ist imstande, Augenblicke besonderer Intensität zu schaffen, wenn er in den Bildern einen Augenblick des Lebens festhält und bei im verweilt mit einer Sprache, die zum Ausdruck echter Poesie werden kann. So kann diese neue Art Kunst viel Wertvolles beitragen zu dem unerschöpflichen Weg der Suche, den der Mensch geht, wenn er die Kenntnis der ihn umgebenden Welt wie auch die seiner inneren Universums erweitert. Natürlich ist es notwendig, den Zuschauern, besonders den jüngeren, $z u$ helfen, daß sie eine Fähigkeit zu kritischem Betrachten der vorgelegten Botschaften in sich ausbilden, damit der Film zum umfassenden, harmonischen Wachstum der Person beitrage.

4. Der Film hat Themen aufgegriffen, die vom Glauben inspiriert waren, und er greift sie auch weiterhin auf. In dieser Hinsicht sind die Heilige Schrift, das Leben Jesu, Marias und der Heiligen, wie auch die Probleme der Kirche unerschöpfliche Quellen für den, der auf der Suche ist nach dem geistigen und religiösen Sinn des Lebens.

So ist es ein Erfolg, daß die Filmkunst oftmals eine erhabene Botschaft zu übermitteln wußte und dazu beigetragen hat, die Respektierung jener Werte zu verbreiten, die das Herz des Menschen bereichern und ohne die es sehr schwierig ist, ein erfülltes und umfassendes Dasein zu leben.

Der Film kann auf diese Weise einen wertvollen Beitrag zur Kultur leisten und der Kirche eine besondere Mitarbeit anbieten. Das wird vor 
allem bedeutsam, während wir uns anschicken, die Schwelle zu einem neuen christlichen Jahrtausend $\mathrm{zu}$ überschreiten. Es wäre mein Wunsch, daß die mit dem Glauben verbundenen Themen immer mit Sachkenntnis und gebührender Achtung behandelt werden.

Auch in den Filmen mit nicht ausgesprochen religiöser Thematik lassen sich echte menschliche Werte finden, eine Auffassung vom Leben und eine Sicht der Welt, die zum Transzendenten hin offen sind. So wird der Austausch zwischen den verschiedenen Kulturen möglich, die sich an dem offenen Fenster zeigen, daß der Film anbietet: Auf diese Weise werden die Entfernungen in der Welt abgekürzt, und das gegenseitige achtungsvolle Verstehen wird begünstigt.

5. Dieses Kommunikationsmittel kann also auch eine pädagogische Funktion übernehmen, die dem Menschen zur Kenntnis der universalen Werte in den verschiedenen Kulturen verhilft und ihn dazu bringt, die berechtigten Unterschiede als Gelegenheit zu wechselseitigem Austausch von Gaben wahrzunehmen.

Der Film ist ein Mittel, das besonders dazu geeignet ist, das unaussprechliche Geheimnis zu erzählen, das die Welt und den Menschen umgibt. Durch Bilder spricht der Regisseur mit dem Zuschauer, überträgt er ihm seine Gedanken, treibt er ihn an, sich Situationen gegenüberzustellen, die das Innere des Menschen nicht unberührt lassen können. Wenn er sich über die Kunst hinaus mit Verantwortungsbewußtsein und Klugheit auszudrücken versteht, kann er seinen spezifischen Beitrag zum großen Dialog zwischen Menschen, Völkern und Kulturen leisten. So wird er in gewisser Weise zum Erzieher, nicht nur für seine Zeigenossen, sondern auch für kommende Generationen, wie es auch bei anderen Kulturschaffenden der Fall ist.

Der Film ist also ein äußerst sensibles Instrument, fähig, in der Zeit jene Zeichen zu lesen, die manchmal dem Blick eines oberflächlichen Beobachters entgehen können. Wenn vom Film guter Gebrauch gemacht wird, kann er zum Wachstum wahrer Menschlichkeit und schließlich zu dem Lob beitragen, das sich vom Geschöpf zum Schöpfer erhebt. Dies ist mein Wunsch für Ihre Tätigkeit. Ich rufe das Licht des Heiligen Geistes auf Thre Mühen im Dienst der Kultur, des Friedens und des Dialogs herab und erteilen Thnen allen und Thren Lieben von Herzen den Apostolischen Segen.

(aus: L'Osservatore Romano Deutsch vom 06.02.1998) 


\section{Chronik}

\section{EUROPA}

\section{Deutschland}

Die Arbeitsgemeinschaft Katholische Presse (AKP) und der Verband Katholischer Verleger und Buchhändler (VKB) streben eine Zusammenarbeit an. Diese Kooperation könnte auf lange Sicht sogar in eine Fusion der beiden Verbände münden. Einen bedeutenden Schritt hin $z u$ einer engeren Verbindung stellt der im Januar 1998 erfolgte Umzug der VKB. Geschäftsstelle in die Bonner Adenauerallee $176 \mathrm{dar}$, wo die AKP schon seit 14 Jahren ihren Sitz hat. AKP und VKB hatten bereits bei einem ersten Kontaktgespräch im vorigen Jahr Möglichkeiten einer Zusammenarbeit erörtert.

Überlegungen zu einer möglichen Kooperation werden innerhalb von Bistumszeitungs-Verlagen und den zuständigen Diözesen im südwestdeutschen Raum angestellt. Dabei handelt es sich um die Kirchenzeitungen für die Bistümer Fulda, Limburg und Mainz, die bereits eine gemeinsame MantelRedaktion unterhalten, sowie um das Trierer Bistumsblatt "Paulinus" und die Speyerer Zeitung "der pilger".

Das Internet und andere OnlineMedien wurden nach einer Repräsentativerhebung der in Nümberg ansässigen Gesellschaft für Konsum-, Markt- und Absatzforschung (GfK) Anfang 1998 von rund 5,8 Millionen Bundesbürgern im Alter zwischen 14 und 59 Jahren genutzt. Knapp eine Million dieser Gruppe würde täglich online gehen - und zwar in der Hauptsendezeit des Fernsehens zwischen 20.00 Uhr und 22.00 Uhr. Die Gesamtzahl der Deutschen, die inzwischen über einen Internet-Zugang verfügen, beziffert die GfK auf zehn Millionen. Unter den regelmäßigen InternetAbfragern haben Frauen einen Anteil von 30 Prozent.

Die TV-Konsumenten im Osten Deutschlands sehen täglich länger fern als die Westdeutschen. $\mathrm{Zu}$ dieser Erkenntnis kommt eine Untersuchung der ZDF-Medienforschung. In Zahlen: Mit 203 Minuten pro Tag habe 1997 der Fernsehkonsum in den neuen Bundesländern um 25 Minuten höher gelegen als in den alten. Die Untersuchung gibt auch Auskunft über die Verweildauer von Kindern vor dem Bildschirm. Die war nach ZDF-Angaben im vergangenen Jahr bei Jungen und Mädchen im Alter zwischen drei und 13 Jahren um vier Minuten auf 95 Minuten gesunken. Hingegen stieg der TV-Konsum bei den Rezipienten ab 14 Jahren um eine Minute auf 196 Minuten. 
Die in Essen ansässige Agentur für internationale Bilddokumentation, foto-present, ist am 31. März 1998 aufgelöst worden. Der Gesellschafter der Agentur, das Bistum Essen, hatte zunächst die Absicht, seiner Gesellschafteranteile aus wirtschaftlichen Gründen zu veräußern. Trotz vorhandener Interessenten kam es jedoch zu keiner Einigung der Übernahme-Modalitäten.

Die evangelische Wochenzeitung "DS - Das Sonntagsblatt" firmiert seit dem Jahreswechsel wieder unter ihrem alten Namen "Deutsches Allgemeines Sonntagsblatt“. Der vor drei Jahren eingeführte kürzere Titel habe sich bei den Lesern nicht durchsetzen können, teilte das Blatt mit. Aus Anlaß ihres 50jährigen Bestehens soll die Wochenzeitung 1998 graphisch neu gestaltet und teilweise farbig gedruckt erscheinen. Die verkaufte Auflage liegt derzeit bei rund 46.000 Exemplaren.

\section{Frankreich}

Mit dem Projekt eines europäischen, katholisch ausgerichteten Sparten-Fernsehkanals hat sich das Europäische Bischöfliche Komitee für Medien (CEEM) auf einer Tagung in Straßburg beschäftigt. Das CEEM berät den Rat der Europäischen Bischofskonferenzen (CCEE) in Medienfragen. Die Schweizer Hauptinitiatoren des religiösen Fernsehkanals, zu denen auch der Altbischof von Lausanne, Genf und
Freiburg/Schweiz, Pierre Mamie, gehört, warben um „moralischen Beistand" der europäischen $\mathrm{Bi}$ schöfe. Der katholisch ausgerichtete Sender mit dem Namen "Eurema" soll in Deutsch, Französisch und Italienisch senden und in Italien, Frankreich, Belgien, Luxemburg, Deutschland, Österreich und der Schweiz ausgestrahlt werden. Initiatoren des Senders sind Schweizer und italienische Privatleute. Geplant sind sechs Stunden Programm täglich.

Die Kulturorganisation der Vereinten Nationen (Unesco) will „Beobachterposten" zur Förderung der „freien Ausübung des Journalismus " in Krisenregionen finanziell unterstützen. Dies kündigte Unesco-Generaldirektor Federico Mayor in Paris an. Derartige „Beobachterposten" könnten sowohl eine Sicherheit für die Regierung wie auch für die Journalisten sein und allen Bürgern zugute kommen, heißt es in der Erklärung. Mayor erinnerte daran, daß 1997 etwa 50 Journalisten bei der Ausübung ihrer Arbeit ums Leben kamen.

Italien

Der italienische katholische Fernsehkanal "SAT 2000" hat seinen Betrieb aufgenommen. Täglich strahlt der Sender der Bischofskonferenz von $10 \mathrm{Uhr}$ bis Mitternacht mehrere Stunden lang kulturelle und religiöse Informationsprogramme sowie Musiksendun- 
gen aus. $\mathrm{Zu}$ empfangen ist er mit einer entsprechenden ParabolAntenne uiber "Hot Bird 2". Eine terrestrische Verbreitung ist durch die Übernahme von Programmteilen durch bereits bestehende lokale Kirchensender geplant. Eine flächendeckende Verbreitung sei jedoch nicht garantiert, meldete Radio Vatikan. Umfassende politische Nachrichtensendungen sind bei "SAT 2000" nicht vorgesehen. Man könne und wolle den bestehenden Stationen hier keine Konkurrenz machen, betonte Dino Boffo, der Direktor der katholischen Zeitung "Avvenire“, der für den Sender zuständig ist. Der Sender wende sich besonders an Familien. Bereits am 26. Januar 1998 hatte der bischofseigene Radiosender „BLU SAT $2000^{\prime \prime}$ seinen Sendebetrieb aufgenommen.

\section{Luxemburg}

Das "Luxemburger Wort", mit mehr als 87.000 Exemplaren mit Abstand die auflagenstärkste $\mathrm{Ta}$. geszeitung des Großherzogtums, feiert in diesem Jahr das 150 jährige Bestehen. Die katholische Zeitung erscheint in der SanktPaulus-Druckerei und erreicht rund 80 Prozent der luxemburgischen Haushalte.

Die erste Nummer war 1848 drei Tage nach der Erklärung der Pressefreiheit durch König-Großherzog Wilhelm II. erschienen. Während des Zweiten Weltkrieges wurde das "Luxemburger
Wort" gleichgeschaltet, Mitarbeiter wurden in Konzentrationslager verschleppt. Nach dem Krieg stieg die Auflage der Zeitung rasant an. Erhebliche Diskussionen um die Ausrichtung der Zeitung endeten 1979 mit dem Beschluß der Synode des Erzbistums Luxemburg, „daß das ,Luxemburger Wort' seine Mission nur erfüllen kann, wenn die journalistische Gestaltungsfreiheit der Redaktion gewahrt" bleibe (Dokumentation vom Festakt im nächsten Heft).

\section{Österreich}

Der Verband Katholischer Publizisten Österreichs hat Maßnahmen gefordert, die "mediale Präsenz und Wirksamkeit der katholischen Kirche in Österreich" abzusichern. Man registriere „mit Besorgnis" jüngste Entwicklungen, die diese Präsenz schwächen, heißt es in einer Eingabe des Verbandes zum „Dialog für Österreich". Es seien - auch finanzielle - Anstrengungen geboten, um wichtige kirchliche Medien und Medieneinrichtungen $\mathrm{zu}$ erhalten und ,in einem schwieriger werdenden Umfeld ihre Wirkmöglichkeiten $z u$ verbessern". Private Initiativen würden dafür kaum ausreichen; "hier ist auch die Hierarchie gefordert", heißt es in der Stellungnahme.

\section{Vatikan}

Das von Papst Johannes Paul II. ausgewählte Thema des kirchlichen Welttages der sozialen Kom- 
munikationsmittel 1998 lautet „In der Kraft des Heiligen Geistes die Hoffnung vermitteln". Der 32. Welttag der Medien wird in den meisten Ländern der Erde am Sonntag vor Pfingsten begangen, der in diesem Jahr auf den 24. Mai fällt. In Deutschland wird der Welttag am 13. September gefeiert. (Wortlaut des Papstwortes siehe DOKUMENTATION)

Papst Johannes Paul II. hat an die Medien appelliert, objektiv über die Fragen des Lebensschutzes und der Menschenwürde zu berichten. Bei seinem Angelusgebet auf dem Petersplatz sagte der Papst, jeder Mensch sei aufgerufen, die Liebe zum Leben zu verbreiten. Insbesondere gelte dies für alle, die in den Massenmedien tätig seien. Positiv verzeichnete Johannes Paul II., daß in den letzten Jahren die Sensibilität für dieses Thema in mancher Hinsicht zugenommen habe. Dennoch seien die schwerwiegenden, gegen das Leben gerichteten Bedrohungen, darunter in erster Linie die Abtreibung, nicht weniger geworden. Daher sei es notwendig, sich dafür einzusetzen, daß die „Kultur des Lebens“ sich gegenüber der „Kultur des Todes“ durchsetze.

"Fides", der seit 70 Jahren bestehende vatikanische Missionsnachrichtendienst, ist einer der traditionsreichsten katholischen Informationsdienste überhaupt. Zum Jubiläum, das Anfang 1998 in Rom begangen wurde, legte $P$.
Bernardo Cervellera (47), der neue Chefredakteur des offiziellen Mitteilungsorgans der römischen Kongregation für die Evangelisierung der Völker, weitreichende Neuerungspläne vor. Er werde im Auftrag des obersten MissionsKoordinators, Kardinal Jozef Tomko, "Fides" "journalistischer und kämpferischer" gestalten.

\section{NORDAMERIKA}

\section{USA}

Leichte Verluste mußte die katholische Presse 1997 hinnehmen. Die Gesamtauflage der 641 Titel sank von 26.790.000 um etwa sechs Prozent auf 25.250.000 Exemplare. Die Auflagenverluste betrafen Bistumspresse und Magazine gleichermaßen. Leichte Anstiege verzeichneten die Newsletters und fremdsprachigen $\mathrm{Kir}$ chenzeitungen, darunter vor allem die in spanischer Sprache.

Die Einschaltquoten bei Kinderprogrammen in den Vereinigten Staaten steigen stetig an. Im Durchschnitt schauten 1997 die US-Kinder zwischen zwei und elf Jahren 312,5 Stunden Kindersendungen, wie die Tagszeitung USA-Today mit Berufung auf eine entsprechende Medienstudie berichtete. Im Jahr 1996 hatten sie noch 295,5 Stunden vor dem Fernseher verbracht. Dabei verfügen 40 Prozent der Sechs- bis Achtjährigen und ein Viertel der Zwei- bis Fünfjährigen über einen 
eigenen Apparat im Schlafzim- gesamt sinkendem Fernsehkonmer. Jungen schauten 53 Stunden sum im Durchschnitt 41 Stunden mehr als Mädchen. Doch auch pro Jahr vor Kindersendungen, Erwachsene verbrachten bei ins- wie die Studie ergab. 